\title{
Modeling and Analysis of Hybrid Systems: A New Approach Integrating Petri Nets and Differential Equations
}

\author{
Gero Nenninger, Volker Krebs \\ Institut für Regelungs- und Steuerungssysteme, Universität Karlsruhe (T.H.), Kaiserstraße 12, \\ D-76128 Karlsruhe, Germany \\ Fax: +(49) 721/608-2707 e-mail: \{nenninge, krebs\}@irs.etec.uni-karlsruhe.de
}

\begin{abstract}
Hybrid technical systems combine discrete-state eventdriven and continuous-state time-driven behavior. In this contribution, a general modeling formalism for such systems is proposed. The discrete subsystem is represented by an interpreted Petri net. In addition to external continuous inputs, discrete variables dependent on the net marking affect the continuous subsystem which is a time-driven state space model. Limit violations of particular continuous state variables as well as external binary inputs are interpreted as events and change the discrete state of the system. In view of the analysis of hybrid technical systems reachability and stability are defined. The modeling method is applied to a mixing tank.
\end{abstract}

\section{Introduction}

Many control engineering methods are based on a mathematical model of the process under investigation. Thus, the available knowledge about the process is used to represent the relevant static and dynamic relationships. This leads to an appropriate model for simulation, analysis, and controller synthesis. It is evident that the granularity has to be adapted to the problem to be solved.

In process and manufacturing engineering as well as in other industrial areas there are processes with a dynamical behavior determined by both, continuous physical dynamics and discrete states with event-driven evolution. The discrete states may originate in the process itself (e.g. a tank is full or empty) or may be caused by a discrete controller generating discrete manipulated variables (e.g. open or closed valves).

An example of such a process is a plant for the supply of production processes with liquid or gas flow. Parallel compressors are disposed in several levels providing a continuous liquid or gas flow on discrete pressure levels. For example, the flow is a continuous quantity typically modeled by differential equations. Discrete states result either from a qualitative view (e.g. start and stop of particular units) or from technological aspects (e.g. discrete positions of valves, safety interlocks). To this day, plants of that type have been controlled by static programming, for the most part. The objective is to develop real-time dynamic control stategies for hybrid plants comprising concurrency.

At first, a hybrid mathematical model will be developed. It takes into account physical variables with their temporal evolution as well as logical relations between discrete states and their transitions.

\section{The Hybrid Dynamical Model (HDM)}

In the past, the study of dynamical systems focused either on time-driven or event-driven dynamical systems. Due to the increasing importance of digital control in industrial applications, several efforts have been made towards modeling formalisms, which combine the two classes of systems [1]. The new Hybrid Dynamical Model (HDM) combines discrete-state event-driven 


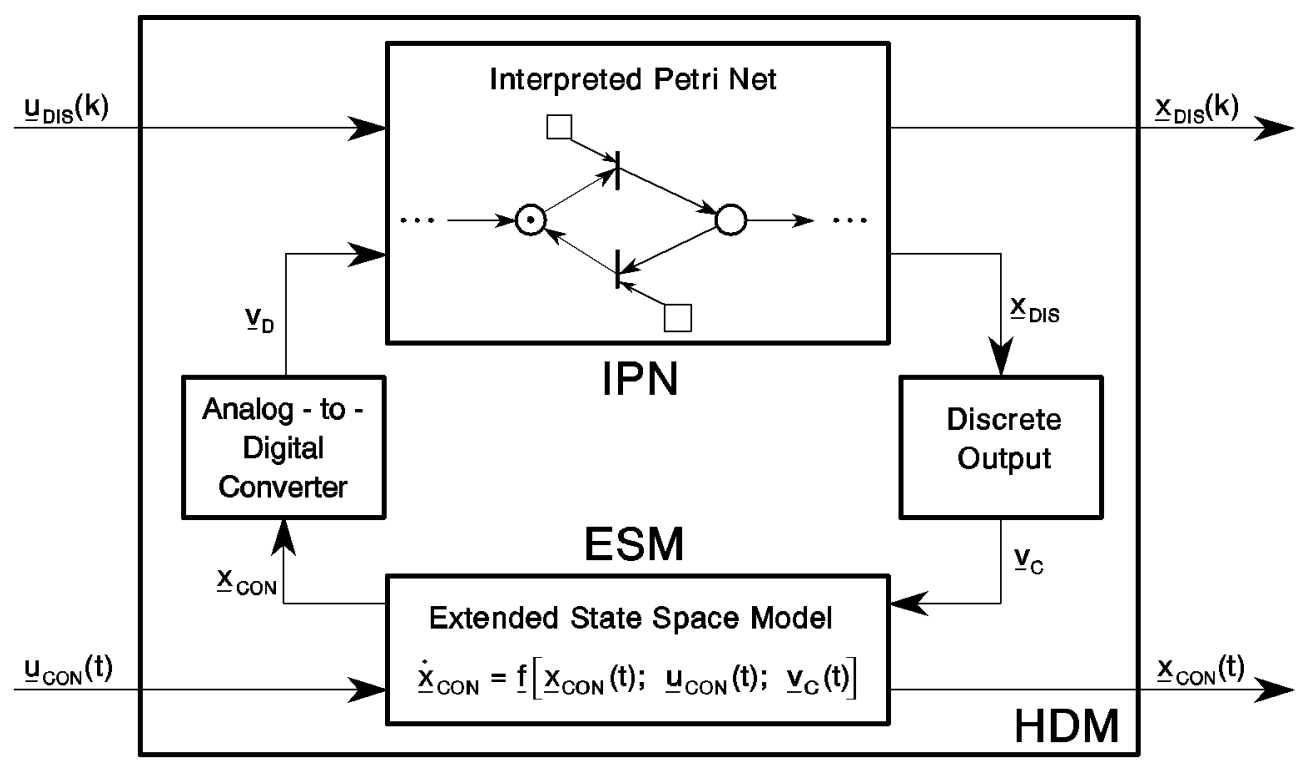

Figure 1: Structure of the Hybrid Dynamical Model (HDM)

components and continuous-state time-driven aspects in a transparent fashion (fig. 1).

The discrete-state part of the system is described by a finite number of states. State transitions are initiated by external influences or internal events. This part is modeled by a Petri net [2]. In contrast to other discrete event models such as finite-state automata, Petri nets allow the modeling of concurrency as well as synchronization. Moreover, they have the ability to modularize a complex system [3]. In interpreted Petri nets (IPN) [4], boolean expressions are assigned to transitions (in fig. 1 depicted as a square with a directed arc to the corresponding transition). They prevent or cause the firing of an enabled transition. These boolean inputs consist of external binary manipulated variables $\underline{u}_{D I S}(k)$ and internal variables $\underline{v}_{D}$. The latter signalize for instance, limit violations of continuous variables:

$$
\underline{v}_{D}=\underline{f}_{D}\left[\underline{x}_{C O N}(t)\right] \in\{0,1\}^{\left|\underline{v}_{D}\right|} .
$$

The dynamic of a Petri net is represented by the firing of transitions. Each firing causes an evolution of the net marking $\underline{x}_{D I S}(k) . k$ stands for the number of discrete state transitions occurred in $[0, t]$. An enabled transition [3] fires immediately iff the corresponding boolean expression is true.

Time-driven continuous dynamics are modeled by differential equations in a state space form [5]. The continuous part of the model depends on $p_{C}$ external continuous inputs $\underline{u}_{C O N}(t)$ and a vector $\underline{v}_{C}$. The instance of a state transition from $\underline{x}_{D I S}(k)$ to $\underline{x}_{D I S}(k+1)$ is marked by $t_{k+1}$. Thus, $\underline{v}_{C}$ is a vector with piecewise constant elements:

$$
\underline{v}_{C}(t)=\underline{f}_{C}\left[\underline{x}_{D I S}(k)\right] \quad, \quad t_{k} \leq t<t_{k+1} .
$$

The usual description of a time-driven system [5] differs from the extended state space model (ESM)

$$
\underline{\dot{x}}_{C O N}(t)=\underline{f}\left[\underline{x}_{C O N}(t) ; \underline{u}_{C O N}(t) ; \underline{v}_{C}(t)\right]
$$

due to the additional input $\underline{v}_{C}$.

If each continuous manipulated variable $u_{C O N}^{(i)}, i=1,2, \cdots, p_{C}$, is bounded,

$$
U^{(i)}=\left\{u_{C O N}^{(i)} \mid u_{C O N, \text { min }}^{(i)} \leq u_{C O N}^{(i)} \leq u_{C O N, \text { max }}^{(i)}\right\}
$$

the vector $\underline{u}_{C O N}(t)$ lies in a $p_{C}$-dimensional hypercube

$$
U_{C}=U^{(1)} \times \cdots \times U^{\left(p_{C}\right)}
$$

of the continuous input space. The whole state of the Hybrid Dynamical Model comprises the set of all discrete and continuous state variables at time $t$ :

$$
\begin{aligned}
x_{H}(k, t):= & \left\{x_{D I S}^{(1)}(k), x_{D I S}^{(2)}(k), \cdots, x_{D I S}^{\left(n_{D}\right)}(k),\right. \\
& \left.x_{C O N}^{(1)}(t), x_{C O N}^{(2)}(t), \cdots, x_{C O N}^{\left(n_{C}\right)}(t)\right\} .
\end{aligned}
$$

In the modeling process, a technical system is divided into subsystems. Each subsystem is described by a Hybrid Dynamical Model. Secondary control is integrated in the individual submodels. A unique Hybrid Dynamical Model of the process is created by means of 
combination of all discrete event parts and all continuous parts.

According to fig. 1, this model has discrete and continuous inputs. Therefore, a hybrid control consists of the simultaneous application of discrete and continuous manipulated variables to the plant. A controller is to influence the process so that the discrete as well as the continuous part behave in a postulated manner.

\section{Analysis of Hybrid Systems}

System analysis is carried out to examine the dynamic properties of an uncontrolled plant or to verify the correctness of a controller with regard to the specifications of the controlled plant. Important characteristics of dynamical systems considered in this paper, are reachability, reconstructability and stability.

In the following, we present definitions of reachability and stability for the Hybrid Dynamical Model and introduce a basic approach to reachability analysis.

In the design phase of plants (e.g. the compressor system in section 1 ) the question arises, whether it is possible to reach a certain set of operating points from an original operating point due to given demands. To investigate this aspect, the concept of reachability is introduced, which means that desired outputs are reached after a finite time interval by appropriate inputs to the system.

\section{Definition 3.1 (Reachability)}

A set $X_{H, e}\left(k_{e}, t_{e}\right)=\left\{x_{H}^{(1)}\left(k_{e}, t_{e}\right), \cdots, x_{H}^{\left(\left|X_{H, e}\right|\right)}\left(k_{e}, t_{e}\right)\right\}$ of hybrid states is reachable from the set $X_{H, 0}(0,0)=$ $\left\{x_{H}^{(1)}(0,0), \cdots, x_{H}^{\left(\left|X_{H, 0}\right|\right)}(0,0)\right\}$, iff there exist valid controls to transfer each state $x_{H} \in X_{H, 0}(0,0)$ to a state $x_{H}^{\prime} \in X_{H, e}\left(k_{e}, t_{e}\right)$ by a finite number of steps $k_{e}$ and within finite time $t_{e}$.

A hybrid control is valid if the discrete input vector is an element of all possible discrete control vectors and the continuous control trajectory does not leave the hypercube $U_{C}$ of the continuous input space.

An approach to examine the reachability of a Hybrid Dynamical Model is to introduce a hybrid reachability graph. Its nodes represent the reachable markings $\underline{x}_{D I S}(k)$ of the interpreted Petri net. The directed arcs connecting the nodes are labelled with the transitions of the Petri net corresponding to the state transitions [2]. Additionally, the continuous subset $X_{C}^{(i)}$ reachable under the discrete state $\underline{x}_{D I S}^{(i)}(k)$ by applying appropriate continuous inputs $\underline{u}_{C O N} \in U_{C}$ is assigned to the individual node.

Stability is the most important property of a dynamical system. With regard to nonlinear systems, the
Lyapunov stability theory is frequently used to analyze the behavior of a system under displacement from an equilibrium point [6].

An operating point of a hybrid technical system represents a hybrid equilibrium point $x_{H, E}$ of the corresponding Hybrid Dynamical Model, if the discrete and continuous states are constant under a constant hybrid control. The following definition describes the behavior of a Hybrid Dynamical Model regarding to a particular equilibrium point.

Definition 3.2 (Stability) $A$ hybrid equilibrium point $x_{H, E}$ with the discrete vector $\underline{x}_{D I S, E}$ and the continuous vector $\underline{x}_{C O N, E}$ is said to be (asymptotically) stable, iff (under application of constant hybrid control variables) there exists a set $X_{H, I}(0,0)$ of initial hybrid states, such that

1. for all $k \geq k_{e} \geq 0: \underline{x}_{D I S}(k)=\underline{x}_{D I S, E}$

and

2. $\underline{x}_{C O N, E}$ is a (asymptotically) stable equilibrium point for $\underline{x}_{C O N}(t)$ in the sense of Lyapunov.

Based on these definitions 3.1 and 3.2, analyzing methods have to be further developed and are currently under investigation.

\section{Modeling of a mixing tank}

A mixing tank (fig. 2) is used as an example to demonstrate the modeling by Hybrid Dynamical Models. $V_{1}$

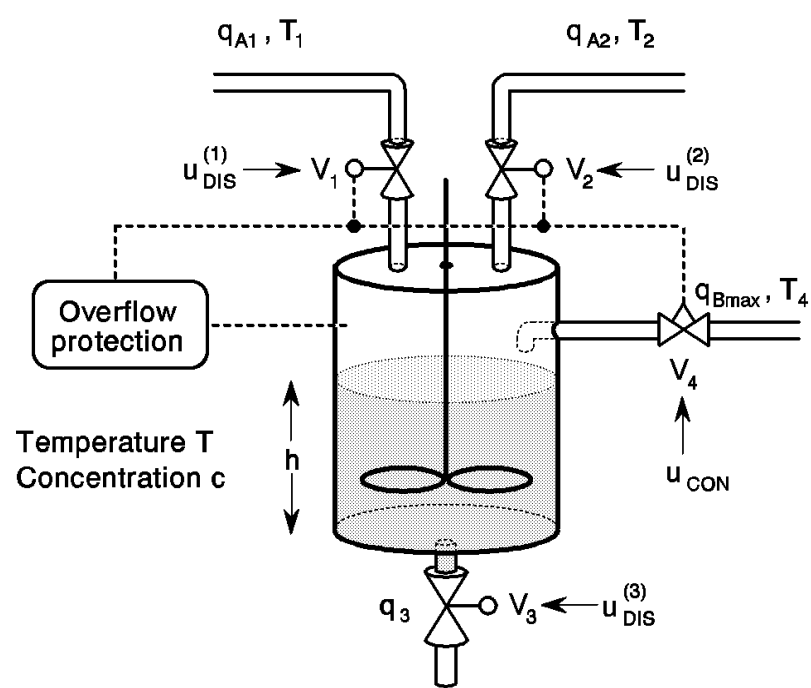

Figure 2: Mixing tank with overflow protection 


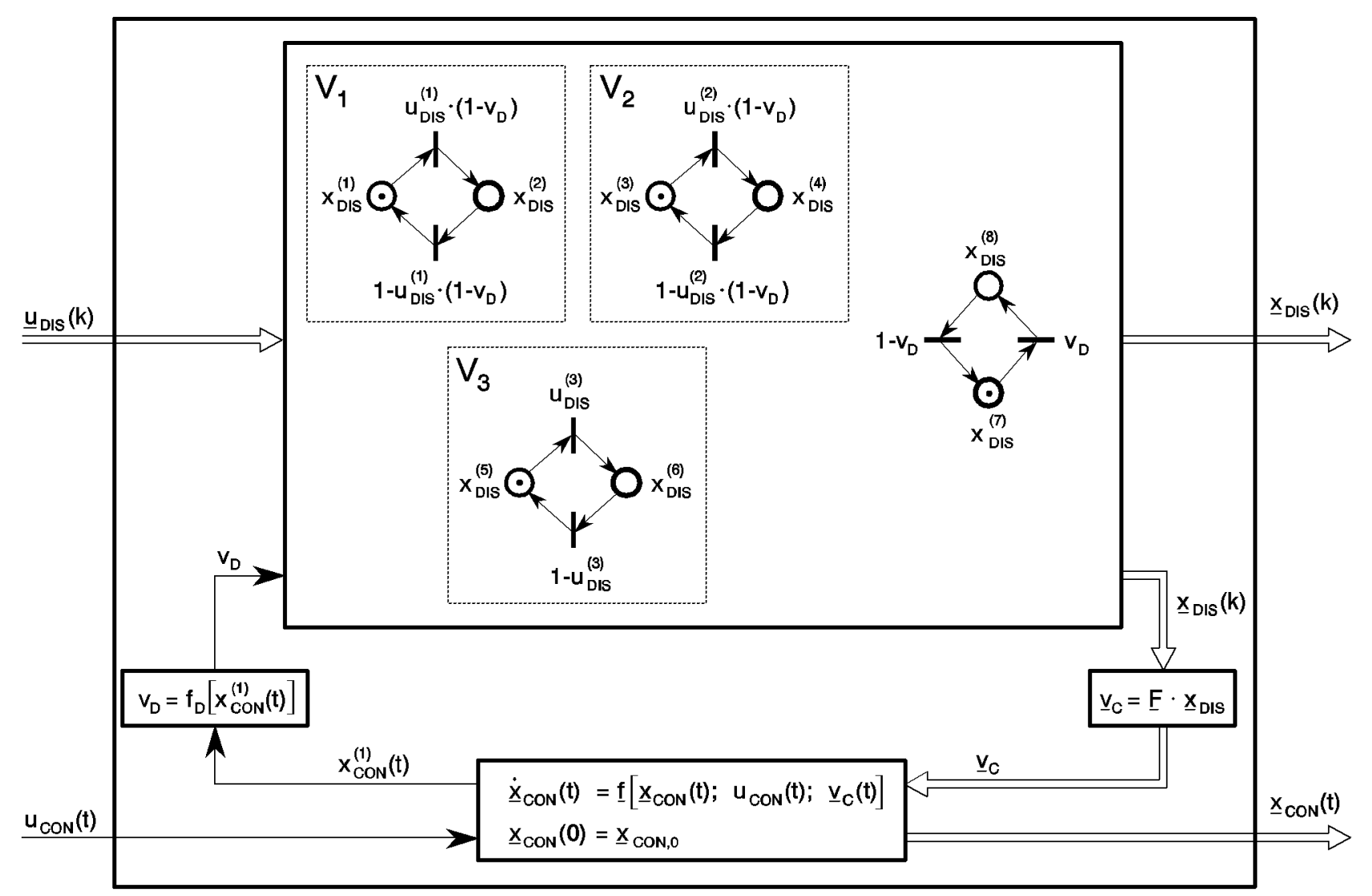

Figure 3: Hybrid Dynamical Model of the uncontrolled mixing tank (fig. 2)

and $V_{2}$ are discrete valves, i.e each of them is either in the discrete state "open" or in the discrete state "closed". Opening $V_{1}$ leads to a constant flow rate $q_{A 1}$ of liquid $\mathrm{A}$ at a temperature $T_{1}$, opening of $V_{2}$ implies a constant flow rate $q_{A 2}$ of liquid $A$ at temperature $T_{2}$, respectively. $V_{4}$ is a continuous valve with linear characteristic and maximal throughput $q_{B \text { max }}$. Corresponding to the value of the manipulated variable $u_{C O N} \epsilon[0,1]$, liquid $\mathrm{B}$ at temperature $T_{3}$ will be fed into the tank. The liquid in the heat-insulated tank is continuously stirred, so that the mixture $A / B$ in the tank is homogeneous. Through the discrete outlet valve $V_{3}$, the mixture $A / B$ can be drained out of the tank with a flow rate $q_{3}$.

All three discrete valves $V_{1}, V_{2}$ and $V_{3}$ are modeled in the Hybrid Dynamical Model of the mixing tank (fig. 3) by Petri nets consisting of two places representing the states "open" and "closed" and two transitions. At the initial marking $\underline{x}_{D I S, 0}$, the places corresponding to the closed valve positions are marked with a token. Additionally, the two places $x_{D I S}^{(7)}$ and $x_{D I S}^{(8)}$ stand for an admissible fluid level and a level equal to an upper limit. The overflow protection, switching off all inflows at a certain fluid level, is described by the variable $v_{D}$ :

$$
v_{D}=f_{D}\left[\underline{x}_{C O N}^{(1)}(t)\right]
$$

As continuous variables, the fluid level $h$ of the mixture $A / B$ in the tank, temperature $T$ of the mixture and concentration $c$ of fluid $B$ in the mixture $A / B$ are of interest. They are integrated in an extended state space model

$$
\underline{x}_{C O N}(t)=\left[\begin{array}{c}
\dot{h} \\
\dot{T} \\
\dot{c}
\end{array}\right]=\underline{f}\left(\underline{x}_{C O N}(t), u_{C O N}(t), \underline{v}_{C}(t)\right)
$$

described in detail in fig. 4.

The vector $\underline{v}_{C}(t)$ represents the influence of the flow rates through the three discrete valves as well as the enabling of $V_{4}$ if the fluid level is lower than its limiting value: 


$$
\begin{aligned}
& \dot{x}_{C O N}^{(1)}= \frac{1}{A_{M}}\left[-a_{1} \cdot \sqrt{x_{C O N}^{(1)}} \cdot v_{C}^{(3)}+v_{C}^{(1)}+v_{C}^{(2)}+q_{B \text { max }} \cdot u_{C O N} \cdot v_{C}^{(4)}\right] \\
& \dot{x}_{C O N}^{(2)}= \frac{1}{A_{M} \cdot x_{C O N}^{(1)}}\left\{\frac{1}{1+\left(\frac{\gamma_{B}}{\gamma_{A}}-1\right) \cdot x_{C O N}^{(3)}} \cdot\left[T_{1} \cdot v_{C}^{(1)}+T_{2} \cdot v_{C}^{(2)}+T_{4} \cdot \frac{\gamma_{B}}{\gamma_{A}} \cdot q_{B \text { max }} \cdot u_{C O N} \cdot v_{C}^{(4)}\right]\right. \\
&\left.-\left(v_{C}^{(1)}+v_{C}^{(2)}+q_{B \max } \cdot u_{C O N} \cdot v_{C}^{(4)}\right) \cdot x_{C O N}^{(2)}\right\} \\
& \dot{x}_{C O N}^{(3)}= \frac{1}{A_{M} \cdot x_{C O N}^{(1)}}\left\{-x_{C O N}^{(3)} \cdot\left(v_{C}^{(1)}+v_{C}^{(2)}\right)+\left(1-x_{C O N}^{(3)}\right) \cdot q_{B \max } \cdot u_{C O N} \cdot v_{C}^{(4)}\right\} \\
& A_{M}: \text { ground area of the mixing tank, } \quad a_{1}=\frac{\sqrt{2 g} \cdot A_{O}}{A_{M}} \text { with } A_{O}: \text { outlet flow area, } \\
& \gamma_{A}, \gamma_{B} \text { : heat capacity of liquid A, B. }
\end{aligned}
$$

Figure 4: Nonlinear extended state space model $\underline{\dot{x}}_{C O N}(t)=\underline{f}\left[\underline{x}_{C O N}(t), u_{C O N}(t), \underline{v}_{C}(t)\right]$

$$
\begin{aligned}
& \underline{v}_{C}(t)= \frac{F}{x_{D I S}} \underline{x}_{D}(k) \\
&=\left(\begin{array}{cccccccc}
0 & q_{A 1} & 0 & 0 & 0 & 0 & 0 & 0 \\
0 & 0 & 0 & q_{A 2} & 0 & 0 & 0 & 0 \\
0 & 0 & 0 & 0 & 0 & 1 & 0 & 0 \\
0 & 0 & 0 & 0 & 0 & 0 & 1 & 0
\end{array}\right) \underline{x}_{D I S}(k) \\
& t_{k} \leq t<t_{k+1} .
\end{aligned}
$$

\section{Conclusion and outlook}

If the combination of event-driven state transitions and continuous dynamics is essential for a technical system, only a hybrid mathematical description adequatly models the process behavior. The Hybrid Dynamical Model introduced in this paper is such a hybrid modeling formalism. An approach to the analysis of reachability was outlined and a stability definition was given. Further research will focus on stability analysis as well as the systematic design of hybrid control strategies.

\section{Acknowledgement}

This work is supported by the Deutsche Forschungsgemeinschaft (DFG) under contract No. Kr 949/7-1.

\section{References}

[1] Labinaz, G.; Bayoumi, M.; Rudie, K.: Modeling and Control of Hybrid Systems: A Survey. IFAC 13th Triennial World Congress 1996, San Fransisco, USA

[2] Abel, D.: Petri-Netze für Ingenieure. SpringerVerlag, Berlin, 1990

[3] Cassandras, C.: Discrete Event Systems: Modeling and Performance Analysis. Aksen Associates and Irwin, 1993

[4] König,R.; Quäck, L.:Petri-Netze in der Steuerungsund Digitaltechnik. Oldenbourg-Verlag, München, 1988

[5] Föllinger, O.: Regelungstechnik. Einführung in die Methoden und ihre Anwendung. Hüthig-Verlag, Heidelberg, 1992

[6] Föllinger, O.: Nichtlineare Regelungen $I$. Oldenbourg-Verlag, München, 1993 